\title{
Efectos de la existencia de capa elástica en los siste- mas de césped artificial de tercera generación y la ve- locidad de carrera sobre la respuesta biomecánica de atenuación de impactos en jugadores de fútbol
}

\author{
A. GARCIA-GALLART, A. ENCARNACIÓN-MARTÍNEZ, A. GALLARDO, \\ J.A. SÁNCHEZ, J. SÁNCHEZ-SÁNCHEZ, I. CHICOY \\ Departamento de Deporte, Universidad Católica de Murcia (UCAM)
}

\begin{abstract}
Resumen
El objetivo del estudio fue comparar la influencia de la capa elástica con otros componentes estructurales sobre la magnitud y atenuación de impactos en diferentes sistemas de césped artificial (SCA) de tercera generación. Para ello, 12 participantes fueron evaluados sobre cuatro SCA, con características estructurales diferentes, mediante un test lineal de carrera a tres velocidades distintas (V1: 3,33 m/s, V2: $4 \mathrm{~m} / \mathrm{s}$ y V3: velocidad máxima) con dos acelerómetros triaxiales situados en tibia y cabeza. El pico de aceleración en tibia fue significativamente menor en SCA4 (sub-base de terreno natural, mayor longitud de fibra y cantidad de relleno) a V3 y menor que SCA1 (fibra de menor longitud y menor cantidad de relleno) y SCA3 (mayor longitud de la fibra, mayor cantidad de relleno y sub-base asfáltica) a V1 y V2. Mientras SCA3, presentó una mayor atenuación con respecto a SCA1 y SCA2 (características estructurales similares a SCA1 y con capa elástica) a V1 y con SCA2 a V2. La capa elástica puede tener una influencia menor que la longitud de la fibra, la cantidad de relleno y la sub-base en la magnitud de los picos de impacto a velocidades máximas y en su capacidad de atenuación a velocidades lentas y moderadas.
\end{abstract}

Palabras clave: Césped artificial, capa elástica, fútbol, impactos, atenuación.

\begin{abstract}
The aim was to compare the influence of elastic layer with other structural components on impact level and attenuation in different artificial turf systems (SCA) of third generation. To this end, 12 participants were evaluated on four SCA with different structural characteristics. A run linear test at three different speeds (V1: 3,33 m/s, V2: $4 \mathrm{~m} / \mathrm{s}$ V3: maximum speed) was used and two triaxial accelerometers were placed in tibia and head. Tibial acceleration peak was significantly lower in SCA4 (sub-base of natural terrain, greater fiber height and infill) to V3 and lower than SCA1 (less fiber height and infill) and SCA3 (greater fibre height and infill and asphaltic sub-base) to V1 and V2. While SCA3, presented a greater attenuation that SCA1 and SCA2 (similar to SCA1 and elastic layer) to V1 and V2 and with SCA2 to V2. Elastic layer may have a minor influence than fibre height, amount of infill and sub-base material on tibial acceleration peak at maximum speeds and attenuation to slow and moderate speeds.
\end{abstract}

Keywords: Artificial turf, shock-pad, football, impact, attenuation.

Correspondencia:

Correspondencia: A. García-Gallart

Departamento de Deporte, Universidad Católica de Murcia (UCAM)

Guadalupe, Murcia, 30107, España

E-mail: agarcia493@alu.ucam.edu 


\section{Introducción}

El fútbol es el deporte más popular del mundo [1], el cual se desarrolló para ser practicado principalmente sobre hierba natural [2]. A pesar de ello, en los años 60 surgió el césped artificial con el objetivo de alzarse como sustituto de la hierba natural [3] debido a su mayor nivel de explotación deportiva, menor coste de mantenimiento, mejor sostenibilidad en instalaciones cubiertas, mejor resistencia a los efectos climáticos y a poder emplearse como medio de explotación económica [3-5].

Se ha demostrado que la superficie es un implemento determinante en la absorción de impactos que se producen con cada contacto del deportista con el suelo [4, 6, 7], los cuales son transmitidos y absorbidos por todo el cuerpo desde el pie hasta la cabeza [8-10] y considerados como uno de los tipos de estrés aplicados a una estructura más importantes [6]. Esta propiedad de atenuación o absorción del pavimento, es la capacidad del mismo para disminuir las fuerzas de impacto que recibe el deportista en diferentes acciones como correr o saltar [11], especialmente importante en deporte de alto impacto como el fútbol, en el cual se combinan fases de alta intensidad con periodos en reposo, desencadenando acciones como carrera, saltos y golpeos con aceleraciones frecuentes y cambios de dirección [12].

Dichas fuerzas de impacto o choques pueden ser pasivas o activas [6], vinculándose a nivel científico un papel determinante de las fuerzas o impactos pasivos en la mayoría de lesiones por sobre uso [13], ya que pueden alcanzar una magnitud de 1 hasta 3 [14] o incluso 5 veces [6] el peso corporal aproximadamente entre los 25 [15], 30 [6] o $50 \mathrm{~ms}$ [16] después del primer contacto con el suelo. Esto, unido a la distancia media recorrida por partido en este deporte, entre 8 y 12 kilómetros por cada jugador [17], y a una posible falta de disipación de los impactos recibidos, puede conllevar a la recepción de elevadas fuerzas que pueden producir sobrecargas y lesiones $[11,18]$. Debido a ello, el choque debe ser atenuado, además de por el riesgo de lesiones que implica, para prevenir la disrupción de los sistemas vestibulares y visuales que se produce por las aceleraciones excesivas en la cabeza $[9,19-23]$.
Las ondas de choque son disipadas internamente por estructuras pasivas y por movimientos activos [18], además de por componentes externos como el calzado y la superficie [10]. En este sentido, el césped artificial se encuentra formado principalmente por un soporte base o baking, relleno (ya sea reciclado o caucho virgen), y fibra, pudiendo incluir en algunos casos una capa elástica o shock-pad [3], donde el impacto es absorbido debido a la compactación de las fibras [24] y por factores determinantes para las propiedades de impacto como son la presencia o no de capa elástica debajo de la superficie y el tipo y cantidad de relleno $[25,26]$. En cuanto a la capa elástica, su inclusión proporciona un mantenimiento de las propiedades rigidez y absorción de impactos durante la vida útil del pavimento, previene el deterioro del mismo por la compactación del relleno y mantiene un rendimiento uniforme a pesar de las posibles variables de la altura de relleno ocasionadas por la práctica deportiva [27]. Por el contrario, supone un mayor coste inicial, no proporciona una mejora en la calidad del juego, al aumentar la elasticidad hace el terreno más lento, dificulta los desplazamientos laterales, aumenta la intensidad de los esfuerzos y se parece menos a las sensaciones de la hierba natural [5].

Debido al gran aumento del número de campos de césped artificial en los últimos años [28], a que la última versión de este tipo de pavimentos, el césped artificial de tercera generación $(3 \mathrm{G})$, presenta comportamientos similares a la hierba natural [3, 29-31], y a que posiblemente las diferencias entre la hierba natural y el césped artificial podrían ser inferiores que las presentes entre diferentes sistemas de césped artificial (SCA) con características estructurales distintas [32, 33], nuestro objetivo fue comparar la influencia de la capa elástica, en la respuesta biomecánica de la interacción jugador-superficie, sobre otros componentes estructurales en la magnitud y la atenuación de las ondas de choque en diferentes SCA de $3 \mathrm{G}$.

\section{Materiales y métodos}

\section{Participantes}

Formaron parte del estudio un total de 12 participantes $(\mathrm{N}=12)$ de sexo varón y una edad 
media de 24,33 \pm 3,73 años, una masa de 73,54 $\pm 5,50 \mathrm{~kg}$, una altura de $178,25 \pm 4,13 \mathrm{~cm}$ y una

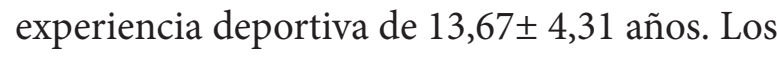
participantes fueron hombres sanos, sin lesiones en el momento de la investigación ni en los 6 meses previos a ella y practicaban fútbol un mínimo de 3 veces a la semana durante los últimos 4 años sobre césped artificial.

Cabe destacar que los participantes fueron informados de las características del estudio y firmaron un consentimiento informado de acuerdo con la Declaración de Helsinki (2013).

\section{Diseño experimental}

Los participantes fueron evaluados sobre 4 SCA de 3G de la marca Mondo (Mondo Ibérica S. A., Zaragoza, España) con características estructurales distintas. Para garantizar un estado de conservación homogéneo, se cumplieron con los requerimientos establecidos en la norma UNE-EN 15330-1:2014 y en estudios previos de referencia [26, 27, 33]. Además, todos tenían una antigüedad inferior a los 5 años, un uso deportivo por debajo de las 35 horas/semana y un mantenimiento específico muy similar. Sus diferencias radicaban en la longitud de la fibra, el tipo de relleno, el tipo de sub-base y la presencia o no de capa elástica o shock-pad (tabla 1).

La prueba consistió en un test lineal de carrera de 20 metros, en una franja de dicha distancia en la parte central del campo en cada uno de los SCA. Con el objetivo de evaluar la magnitud y la transmisibilidad del impacto en la interacción jugador-superficie, se detectaron los picos de aceleración en tibia (PAT) y cabeza (PAC) y se calculó la magnitud de atenuación del impacto (MAI), es decir, cuanto porcentaje de aceleración registrado en la tibia es atenuado hasta que llega a la cabeza (figura 1). Se

\begin{tabular}{|c|c|c|c|c|}
\hline SCA & Fibra & Relleno & Sub-base & Capa elástica \\
\hline 1 & RM 3NX - 45 mm & $\mathrm{A}\left(20 \mathrm{Kg} / \mathrm{m}^{2}\right)+\operatorname{Ecofill}\left(9 \mathrm{Kg} / \mathrm{m}^{2}\right)$ & AS & No \\
\hline 2 & RM 3NX - 45 mm & $\mathrm{A}\left(20 \mathrm{Kg} / \mathrm{m}^{2}\right)+\operatorname{Ecofill}\left(9 \mathrm{Kg} / \mathrm{m}^{2}\right)$ & AS & Sí (17 mm) \\
\hline 3 & RM 4NX - 60 mm & $\mathrm{A}\left(16 \mathrm{Kg} / \mathrm{m}^{2}\right)+\mathrm{SBR}\left(17 \mathrm{Kg} / \mathrm{m}^{2}\right)$ & AS & No \\
\hline 4 & RM 4NX - 60 mm & $\mathrm{A}\left(16 \mathrm{Kg} / \mathrm{m}^{2}\right)+\mathrm{SBR}\left(17 \mathrm{Kg} / \mathrm{m}^{2}\right)$ & $\mathrm{TN}$ & No \\
\hline
\end{tabular}

Tabla 1. Características estructurales de los sistemas de césped artificial.

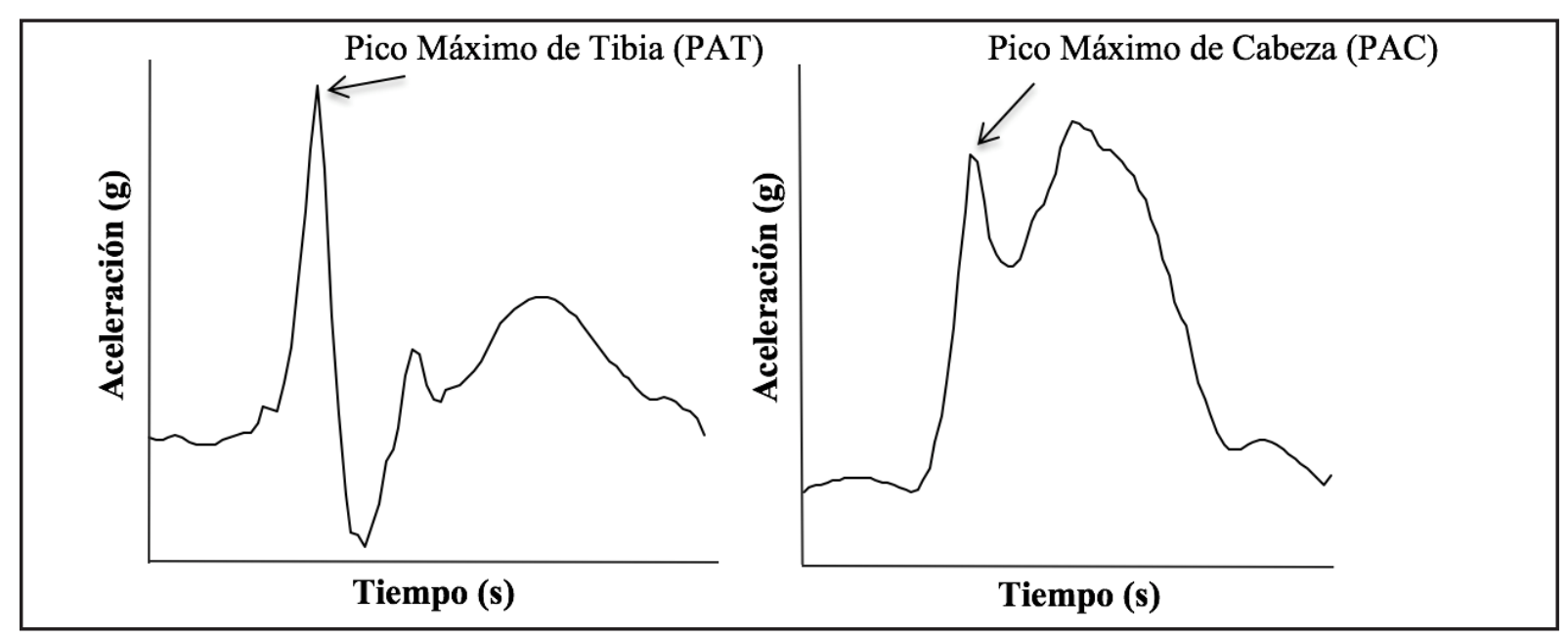

Figura 1. Localización de pico máximo en señal de tibia (izquierda) y cabeza (derecha). 
empleó un sistema de acelerometría inalámbrica de baja masa [8] y de tipo triaxial (Blautic, Valencia, España), con una frecuencia de muestreo de $300 \mathrm{~Hz}$ y un rango de medición de hasta $16 \mathrm{~g}$. Los acelerómetros fueron colocados sobre prominencias óseas en la parte medial y anterodistal de la tibia derecha y en el hueso frontal del cráneo de los participantes [34] con cinta de doble cara a la piel y se reforzó su sujeción con cintas de neopreno ajustando la presión hasta el límite de confort de los participantes [35].

Tras la instrumentación y posterior calibración del sistema de acelerometría, los participantes llevaron a cabo un calentamiento previo al test de 12 minutos, compuesto por ejercicios de carrera continua moderada, estiramientos balísticos de la musculatura del miembro inferior y series de carrera en creciente intensidad, donde al finalizar el mismo, probaron los ritmos de carrera establecidos excepto para la condición de velocidad máxima, hasta que se sintieron confortables con las condiciones de estudio.

El test lineal fue realizado mediante tres velocidades distintas de carrera, controladas mediante un sistema de células fotoeléctricas (Microgate, Bolzano, Italia) colocadas en los 5 metros centrales del pasillo de carrera. La primera velocidad (V1) fue marcada a 3,33 $\mathrm{m} / \mathrm{s}[36,37]$, siendo la velocidad más utilizada en la mayoría de estudios consultados, mientras la segunda velocidad (V2) fue de $4 \mathrm{~m} / \mathrm{s}$ [9, 38-41], considerada como alta y empleada en estudios consultados. Además, al ser en la cantidad de trabajo a alta intensidad y sprint, la cual representa entre el 1 y el $12 \%$ del tiempo total de un partido [42] manifestándose en rangos de 10 a 30 metros o de 2 a 4 segundos [43], donde se encuentran los momentos decisivos para el resultado del mismo [44], determinamos una tercera velocidad (V3) que fuera la máxima posible en 20 metros.

Cabe destacar que el orden de las velocidades fue aleatorizado, se llevaron a cabo tres repeticiones para cada condición de velocidad, aceptando por válidas aquellas repeticiones con una desviación en la velocidad del $\pm 5 \%$ con respecto a la velocidad fijada para cada condición, debiendo repetir el intento si no se ajustaba a dichos límites. En cuanto a la señal de acelerometría, fue registrada durante 5 segundos, los cuales coincidían con el paso por la zona central donde estaban ubicadas las fotocélulas con el objetivo de garantizar la estabilidad de la velocidad y evitar los momentos de aceleración inicial y de desaceleración final de los test [8]. Además, se mantuvo un descanso de 2 minutos entre cada repetición para evitar la fatiga y las pruebas fueron realizadas con un descanso mínimo de 48 horas entre ellas para evitar los efectos de la fatiga.

\section{Análisis estadístico}

Terminada la toma de datos, los datos fueron ordenados y la señal de acelerometría fue procesada aplicando un filtro de paso bajo Butterworth de segundo orden de $60 \mathrm{~Hz}$ [9]. Tras ello, las variables de estudio fueron calculadas a través de una rutina creada exclusivamente para la extracción de las mismas en el programa MATLAB R2013b (MathworksInc, Natick, MA).

Una vez extraídos los datos de las variables de interés, se realizó un análisis estadístico mediante el programa SPSS 19 (SPSS Inc., an IBM Company, Chicago, IL, USA). Por un lado, se llevó a cabo un análisis exploratorio donde se comprobó la normalidad de la muestra mediante la prueba Shapiro-Wilk y la homogeneidad de las varianzas mediante el test de Levene. Se realizó un ANOVA de medidas repetidas de dos factores para determinar las existencia de diferencias entre SCA y entre las velocidades de carrera. Se aplicó una prueba post-hoc de Bonferroni o Games-Howell, según la distribución normal o no de los datos, para determinar las diferencias específicas entre SCA y entre velocidades. Se estableció un valor de $\mathrm{p}<0.05$ como valor significación estadística. Por último, fueron calculados los intervalos de confianza (CI 95\%) de dichas diferencias y el tamaño del efecto a través de la $\mathrm{d}$ de Cohen (d), donde los valores entre 0 y 0.2 representaban un tamaño del efecto pequeño, moderado entre 0.5 y 0.8 y alto con valores superiores a 0.8 [45]. 
capa elástica (presente en SCA2) a velocidades lentas y moderadas, podría suplirse añadiendo una mayor longitud de fibra, cantidad de relleno y una sub-base de terreno natural, presentes en SCA4, ya que el efecto de la capa elástica a velocidades submáximas es diluido a velocidades máximas, donde la presencia de dicha sub-base natural en SCA4 podría ser ventajosa para la eficiencia de los componentes de impacto a sprint. El resto de SCA presentaban una sub-base asfáltica, lo que añadido a la menor longitud de la fibra en SCA1 y SCA2 y la menor cantidad de relleno, en base a la evidencia sobre el asfalto, podrían tener una capacidad de atenuación menor, ya que presenta unos choques más duros y no absorbe el impacto con tanta eficacia como superficies más blandas [46, 47] como en el terreno natural de SCA4, presentando una excelente absorción $[46,47]$.

\section{$P A C$}

No se encontraron diferencias significativas en PAC, aspecto que concuerda con evidencias previas, ya que el sistema de protección corporal mantiene constantes los impactos que llegan a cabeza para prevenir la disrupción de los sistemas vestibulares y visuales que se podrían producir por las aceleraciones excesivas en la cabeza [9, 19-23].

\section{MAI}

Artículos previos han descrito una mayor absorción del impacto en SCA de 3G con capa elástica y una cantidad de relleno aumentada $[25,26]$. Cierto es que estos mismos autores emplearon calzado multitaco, el cual presenta mayor grosor en la suela, que parece aumentar la absorción del impacto. Por otro lado, en otro estudio [33] compararon cuatro SCA con características estructurales diferentes, encontrando un porcentaje mayor de reducción de fuerzas $(69.83 \pm 1.18 \%)$ en SCA con sub-base de graba compacta y capa elástica de $23 \mathrm{~mm}$ de espesor, relleno de arena $\left(15 \mathrm{~kg} / \mathrm{m}^{2}\right)$ y caucho SBR $\left(8 \mathrm{~kg} / \mathrm{m}^{2}\right)$ y una altura de fibra de polietileno de $45 \mathrm{~mm}$. Éste campo, denominado en el artículo original como Sistema 2, se diferenciaba del Sistema 4 en la sub-base asfáltica y en el menor espesor de la capa elástica $(12 \mathrm{~mm}) \mathrm{de}$ este último, con un porcentaje de reducción de $48.07 \pm 3.67 \%$. Mientras los Sistemas 1 y 3, poseían una altura de la fibra mayor (60 mm), mayor cantidad de arena $\left(20 \mathrm{~kg} / \mathrm{m}^{2}\right)$ y caucho SBR $\left(13 \mathrm{~kg} / \mathrm{m}^{2}\right)$, no incorporaban capa elástica y la sub-base era de graba compacta y asfalto respectivamente, manifestando un porcentaje de reducción de $51.30 \pm 2.52 \%$ y $60,10 \pm 2.04 \%$ [33].

En nuestra investigación, SCA3 permite atenuar en mayor porcentaje el impacto durante velocidades lentas en comparación con SCA1 y SCA2, y en velocidades moderadas con respecto a SCA2. Estos resultados, debido a los componentes estructurales de SCA3, para la muestra de jugadores y SCA seleccionados no concuerdan con las investigaciones precedentes que concluyen que la presencia de capa elástica deriva en una mayor atenuación de los impactos $[25,26,33]$.

Nuestros resultados deben tomarse con cautela, ya que Sánchez-Sánchez, et al. [27] sugieren tras un análisis de regresión lineal, que la capa elástica tiene un papel significativo en las propiedades mecánicas de los SCA, siendo más influyente que la sub-base y el año de evaluación. Estos autores muestran en un análisis longitudinal que los SCA con capa elástica manifestaban una reducción de fuerzas significativamente mayor que los SCA que carecían de la misma, debido a que compensa la perdida de elasticidad provocada por la reducción en la fibra con el paso del tiempo. Además, evidencian el papel de la capa elástica en la conservación de las propiedades del pavimento y en el aumento de la vida útil de los mismos, ya que reduce los efectos de uso. Cabe destacar que estas posibles diferencias entre los resultados de estos investigadores [27] y los presentes en nuestro estudio puedan deberse a las diferentes metodologías empleadas, ya que estos autores evaluaron el comportamiento mecánico de SCA de $3 \mathrm{G}$ con una antigüedad suficiente para mostrar el deterioro derivado de un uso deportivo. Mientras en nuestra investigación, se registraron parámetros biomecánicos de la interacción jugadorsuperficie en SCA de $3 \mathrm{G}$ que presentaban un estado de conservación homogéneo, así como una antigüedad, uso deportivo y mantenimiento similar. Por ello, cabe matizar que la presencia de capa elástica en SCA de 3G de una anti- 
güedad inferior a 5 años y un mantenimiento y uso similares, no proporciona una capacidad de atenuación de impactos mayor que otros sistemas que carezcan de ella. A pesar de ello, habría que analizar el efecto de la capa elástica en estos SCA sobre las características de atenuación de impactos cuando, por la antigüedad y el uso, se haga evidente el desgaste de sus componentes.

Por otro lado, la mayor cantidad de relleno en SCA3 y SCA4 (SBR) con respecto a SCA1 y SCA2 (virgen termoplástico Ecofill), podría afirmar el papel determinante de este componente en la atenuación de impactos como reflejan otras investigaciones $[25,26]$, no afectando a los resultados que el tipo de relleno sea distinto [48]. Esto, unido a la fibra de mayor longitud (60 mm en SCA3 y SCA4) puede convertir estos pavimentos en superficies más blandas, lo que favorecería su comportamiento ante la magnitud y la transmisión de impactos $[46,47]$.

\section{Conclusión}

Los resultados mostrados en nuestro estudio reflejan, para la muestra y los sistemas de césped artificial seleccionados, que la presencia de capa elástica en sistemas de césped artificial de tercera generación puede tener una influencia menor en la respuesta biomecánica de la interacción jugador-superficie que otros componentes estructurales como la longitud de la fibra, la cantidad de relleno y el material de la sub-base tanto en la magnitud de los picos de impacto en tibia a velocidades máximas, como en la capacidad de atenuación de dichos impactos a velocidades lentas y moderadas en sistemas de césped artificial de tercera generación con una antigüedad inferior a 5 años, un uso deportivo inferior a 35 horas/semana, un estado de conservación homogéneo y mantenimiento similar.

\section{Agradecimientos}

Esta investigación fue llevada a cabo en la Universidad Católica de Murcia y financiada gracias a una ayuda del plan propio de investigación PMAFI 21/14.

\section{Bibliografía}

1. Reilly T, Bangsbo J, Franks A. Anthropometric and physiological predispositions for elite soccer. J Sport Sci 2000;18(9):669-683.

2. Ekstrand J, Nigg BM. Surface-related injuries in soccer. Sport Med 1989;8(1):56-62.

3. Burillo P, Felipe JL, Gallardo A, Gallardo L, Sanchís M, Gude R, et al. La revolución del pavimento en el fútbol. El césped artificial. Valencia: Servicio de Publicaciones de la Universidad de Valencia, 2010.

4. Nigg BM, Yeadon MR. Biomechanical aspects of playing surfaces. J Sport Sci 1987;5(2):117-145.

5. Párraga JA, Sánchez A. Estudio comparativo sobre los costes de mantenimiento y rentabilidad económica, social y deportiva entre campo de fútbol con pavimentos de césped natural y artificial en el ámbito universitario. Eur J Hum Mov 2002;8:21-46.

6. Hreljac A. Impact and overuse injuries in runners. Med Sci Sports Exerc 2004;36(5):845-849.

7. Nigg BM, Cole GK, Brüggemann G. Impact forces during heel-toe running. J Appl Biomech 1995;11:407-432.

8. Encarnación-Martínez A, Pérez-Soriano $P$, Llana-Belloch S. Differences in Ground Reaction Forces and Shock Impacts Between Nordic Walking and Walking. Res Q Exerc Sport 2015;86(1):94-99.

9. Gruber AH, Boyer KA, Derrick TR, Hamill J. Impact shock frequency components and attenuation in rearfoot and forefoot running. J Sport Health Sci 2014;3(2):113-121.

10. Mercer JA, Bates BT, Dufek JS, Hreljac A. Characteristics of shock attenuation during fatigued running. J Sport Sci 2003;21(11):911-919.

11. Sanchis M, Rosa D, Pérez V, Lahuerta R, Alcántara E. Introducción a los pavimentos deportivos. En: Pérez-Soriano P, Llana-Belloch S, editores. Biomecánica Básica Aplicada a la Actividad Física y el Deporte. 1a ed. Badalona: Paidotribo, 2015. p. 313-328.

12. Sánchez-Sánchez J, García-Unanue J, Jiménez-Reyes P, Gallardo A, Burillo P, Felipe JL, et al. Influence of the Mechanical Properties of Third-Generation Artificial Turf Systems on Soccer Players' Physiological and Physical Performance and Their Perceptions. PLOS ONE 2014; 9(10):e111368.

13. Benson LC, O'Connor KM. The Effect of Exertion on Joint Kinematics and Kinetics During Running Using a Waveform Analysis Approach. J Appl Biomech 2015;31:250-257.

14. De Wit B, De Clercq D, Aerts P. Biomechanical analysis of the stance phase during barefoot and shod running. J Biomech 2000;33(3):269-278.

15. Hasegawa $H$, Yamauchi $T$, Kraemer WJ. Foot 
strike patterns of runners at the $15-\mathrm{km}$ point during an elite-level half marathon. J Strength Cond Res 2007;21(3):888-893.

16. Wright IC, Neptune RR, van Den Bogert AJ, Nigg BM. Passive regulation of impact forces in heel-toe running. Clin Biomech 1998;13(7):521531.

17. Bangsbo J, Mohr M, Krustrup P. Physical and metabolic demands of training and match-play in the elite football player. J Sport Sci 2006;24(7):665674.

18. Blackah N, Bradshaw EJ, Kemp JG, Shoushtarian M. The Effect of Exercise-Induced Muscle Damage on Shock Dissipation during Treadmill Running. Asian J Sports Med 2013;10(1):16-30.

19. Derrick TR, Hamill J, Caldwell GE. Energy absorption of impacts during running at various stride lengths. Med Sci Sports Exerc 1998;30:128135.

20. Edwards WB, Derrick TR, Hamill J. Musculoskeletal attenuation of impact shock in response to knee angle manipulation. J Appl Biomech 2012;28:502-510.

21. Pozzo T, Berthoz A, Lefort L, Vitte E. Head stabilization during various locomotor tasks in humans. Ii. Patients with bilateral peripheral vestibular deficits. Exp Brain Res 1991;85:208-217.

22. Lafortune MA, Lake MJ, Hennig EM. Differential shock transmission response of the human body to impact severity and lower limb posture. J Biomech 1996;29:1531-1537.

23. Hamill J, Derrick TR, Holt KG. Shock attenuation and stride frequency during running. Hum Mov Sci 1995; 14:45-60.

24. Anderson LJ, Fleming PR, Ansarifar A. Shock Absorbing Layers for Synthetic Sports Pitches. Eng Sport 2004;5:509-516.

25. McGhie D, Ettema G. Biomechanical analysis of surface-athlete impacts on third-generation artificial turf. Am J Sports Med 2013;41(1):177-185.

26. Alcántara E, Gámez $J$, Rosa $D$, Sanchis $M$. Analysis of the influence of rubber infill morphology on the mechanical performance of artificial turf surfaces for soccer. J Sports Eng Technol 2009;223:1-9.

27. Sánchez-Sánchez J, Felipe JL, Burillo P, del Corral J, Gallardo L. Effect of the structural components of support on the loss of mechanical properties of football fields of artificial turf. J Sport Eng Technol 2014; 228(3):155-164.

28. Burillo P, Gallardo L, Felipe JL, Gallardo AM. Mechanical assessment of artificial turf football pitches: The consequences of no quality certification. Sci Res Essays 2012;7(28):2457-2465.

29. Ekstrand J, Timpka T, Hägglund M. Risk of injury in elite football played on artificial turf versus natural grass: a prospective two-cohort study. Brit J Sport Med 2006;40:975-980.

30. Fuller CW, Dick RW, Corlette J, Schmalz R.
Comparison of the incidence, nature and cause of injuries sustained on grass and new generation artificial turf by male and female football players. Part 1: match injuries. Brit J Sport Med 2007;41(1):20-26.

31. Fuller CW, Dick RW, Corlette J, Schmalz R. Comparison of the incidence, nature and cause of injuries sustained on grass and new generation artificial turf by male and female football players. Part 2: training injuries. Brit J Sport Med 2007;41(1):27-32.

32. Potthast $W$, Verhelst $R$, Hughes $M$, Stone $K$, De Clercq, D. Football-specific evaluation of playersurface interaction on different football turf system. Sport Technol 2010;3(1):5-12.

33. Sánchez-Sánchez J, García-Unanue J, JiménezReyes P, Gallardo A, Burillo P, Felipe JL, et al. Influence of the Mechanical Properties of Third-Generation Artificial Turf Systems on Soccer Players' Physiological and Physical Performance and Their Perceptions. PLOS ONE 2014;9(10):e111368.

34. Lafortune MA, Hennig EM. Contribution of angular motion and gravitation to tibiat acceleration. Med Sci Sports Exerc 1991;23(3):360-363.

35. Derrick T, Dereu D, McLean S. Impacts and kinematic adjustments during an exhaustive run. Med Sci Sports Exerc 2002;34:998-1002.

36. Bahlsen HA, Nigg BM. Influence of attached masses on impact forces and running style in heel-toe running. Int J Biomech 1987;3:264-275.

37. Hardin EC, van den Bogert AJ, Hamill J. Kinematic adaptations during running: effects of footwear, surface, and duration. Med Sci Sports Exerc 2004;36(5):838-844.

38. Boyer KA, Nigg BM. Muscle activity in the leg is tuned in response to impact force characteristics. J Biomech 2004;37(10):1583-1588.

39. Hamill J, Russell EM, Gruber AH, Miller R. Impact characteristics in shod and barefoot running. Footwear Sci 2011;3(1):33- 40.

40. Nigg BM, Bahlsen HA. Influence of heel flare and midsole construction on pronation, supination, and impact forces for heel-toe running. Int J Sport Biomech 1988;4(3):205-219.

41. Shorten MR, Winslow DS. Spectral analysis of impact shock during running. Int J Sport Biomech 1992;8:288-304.

42. Di Salvo V, Baron R, González-Haro C, Gormasz C, Pigozzi F, Bachl N. Sprinting analysis of elite soccer players during European Champions League and UEFA Cup matches. J Sport Sci 2010;28(14):1489-1494.

43. Buchheit M, Bishop D, Haydar B, Nakamura FY, Ahmaidi S. Physiological responses to shuttle repeated-sprint running. Int J Sport Med 2010;31(6):402-409.

44. Spencer M, Bishop D, Dawson B, Goodman C. Physiological and metabolic responses of repeatedsprint activities. Sport Med 2005;35(12):1025-1044. 
46. Cohen J. Statistical Power Analysis for the Behavioral Sciences. NuevaYork: Academic Press, 1969.

47. Glover B, Glover SL. Manual del corredor de competición. Badalona: Editorial Paidotribo, 2005.

48. Jutel A. Descubre y practica la carrera a pie. Barcelona: Inde Publicaciones, 2000.

49. Meijer K, Dethmers J, Savelberg HH, Willems PJB, Wijers B. Biomechanical analysis of running on third generation artificial soccer turf. En:
Haake S, Moritz EF, editores. The Engineering of Sport 6. Vol 1. New York: Springer, 2006:29-34. siol 1997;75:233-238.

50. Burns J, Landorf KB, Ryan MM, Crosbie J, Ouvrier RA. Interventions for the Prevention and Treatment of Pes Cavus. 2007;D006154.

51. Burns J, Crosbie J, Ouvrier R, Hunt A. Effective Orthotic Therapy for the Painful Cavus Foot. J Am Podiatr Med Assoc 2006;96:205-211.

52. Carmona Gómez PM. Influencia De La Información Termográfica Infrarroja En El Protocolo De 